\title{
Effects of 2'-Demethoxy-2' -propoxygriseofulvin on Microtubule Distribution in Chinese Hamster V79 Cells
}

\author{
Taiko Oda
}

Received: August 26, 2005 / Accepted: January 11, 2006

(C) Japan Antibiotics Research Association

\begin{abstract}
Griseofulvin may combine with tubulin as a component of microtubules and, at high doses, inhibits microtubule formation in mammalian cells. In this study, the author examined the effects of three various 2 'substitutive derivatives of griseofulvin on microtubule distribution in Chinese hamster V79 cells. The results showed that 2 '-demethoxy-2' propoxygriseofulvin was the strongest inhibitor of a normal cytoplasmic microtubule network, unlike findings in previous in-vitro studies.
\end{abstract}

Keywords griseofulvin, 2'-demethoxy-2'-propoxygriseofulvin, microtubule, Chinese hamster V79 cells

\section{Introduction}

Griseofulvin (1) was isolated from Penicillium griseofulvum DICK and as a "curling factor" from $P$. janczewskii Zal., and its structure and biological properties have been reported [1]. We used deuterium as a stable isotopic tracer; a biosynthetic study of $\mathbf{1}$ and stereo-specific microbial transformation for $\mathbf{1}$ were reported previously [2 5]. 1 has been used clinically to treat tinea pedis (athlete's foot). Recently, studies aiming at application for cancer therapy have been reported $[6,7]$.

The mechanism of action of $\mathbf{1}$ involves alterations of microtubules and at high concentrations the drug has effects in human cells. 1 may bind to tubulin and, like colchicines and vinblastin, when combined with tubulin as a component of microtubules, it inhibits microtubule formation $[8,9]$. Using the viscosity detection method to

T. Oda: Department of Pharmaceutical Sciences, Kyoritsu College of Pharmacy, Shibakoen 1-chome, 5-30, Minato-ku, Tokyo 105-8512, Japan assess the polymerization and depolymerization of tubulin, it was found that $\mathbf{1}$ and derivatives $\mathbf{2} \sim \mathbf{4}$ were equally potent $[10,11]$.

In this study, the effects of 29-demethoxygriseofulvin (2) and its 29-substituted derivatives ( 3 and 4 ) on microtubule distribution in the Chinese hamster V79 cell were examined. Results show that 2'-demethoxy-2'propoxygriseofulvin (3) has the strongest inhibiting activity on microtubule formation of the test compounds.

\section{Materials and Methods}

\section{Materials}

Griseofulvin (1) was purchased from Nippon Kayaku Co., Ltd. 2'-Demethoxygriseofulvin (2), 2'-demethoxy$2^{\prime}$-propoxygriseofulvin (3) and 2'-demethoxy-2'methylgriseofulvin (4) were prepared as described previously [12 14]. These structures are shown in Fig. 1. Fetal bovine serum (FBS) was obtained from GIBCO after lot-checking.

\section{Cell Culture}

The cell culture was essentially the same as that described in the previous paper [15]. Chinese hamster V79 cells were grown in monolayer culture in Eagle's minimum essential medium (MEM, Nissui Pharmaceutical Co., Ltd., Tokyo) with $10 \%$ heat-inactivated FBS.

\section{Relative Plating Efficiency}

This method was essentially the same as that described in a previous paper [15], except that 1, 2, 3 and $\mathbf{4}$ were used as the samples. The relative plating efficiencies in the presence of different concentrations of the samples were determined as the ratio of the number of colonies at a given 
<smiles>[R]C1=CC(=O)[CH]C(C)[Y]12oc1c(Cl)c(OC)cc(OC)c1c2=O</smiles>

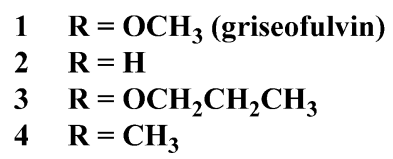

Fig. 1 Structures of griseofulvin and its derivatives.

drug concentration to that obtained in the control culture in the absence of any samples. Two hundred cells were seeded on $60 / 15-\mathrm{mm}$ Petri dishes in $4 \mathrm{ml}$ of MEM with $10 \%$ FBS. At 24 hours after seeding, the samples dissolved in DMSO ( $4 \mu \mathrm{l})$ were added to the culture for 48 hours. Then, the medium was replenished, and the culture was continued for 48 hours without drugs. The dishes were fixed with methanol and stained with 7\% Giemsa solution. The number of colonies ( $>50$ cells/colony) was counted under a dissecting microscope.

\section{Indirect Immunofluorescence}

All of the experimental conditions were essentially the same as described in the previous paper [15], except that $\mathbf{1}$, 2, 3 and $\mathbf{4}$ were used as the samples. Cells were fixed, treated with $1 \%$ Triton X-100 in phosphate-buffered saline (PBS), preincubated for 30 minutes with $2 \%$ dry milk in PBS at room temperature, and then incubated for 1 hour with $20 \mu \mathrm{l}$ per well of mouse monoclonal anti- $\beta$ tubulin antibody $(10 \sim 20 \mu \mathrm{g} / \mathrm{ml}$; Amersham Laboratories, Buckinghamshire, U.K.) at $37^{\circ} \mathrm{C}$. Following three 5 minutes rinses in PBS containing 2\% dry milk, the cells were further incubated for 1 hour with fluorescein isothiocyanate (FITC)-conjugated sheep anti-mouse IgG1 (125 $\mu \mathrm{g} / \mathrm{ml}$; Binding Site Ltd., Birmingham, U.K.) at $37^{\circ} \mathrm{C}$. Following a 15 -minutes rinse in PBS containing 2\% dry milk, and then in PBS, the wet slides were mounted in FA mounting fluid at pH 9 (Difco Laboratories, Detroit, MI). The slides were examined and photographed with Fujicolor Super HG 400 (ASA 400) film under a 100× Olympus Dapo UV lens, using an Olympus BHS-RFK fluorescence microscope with an Olympus PM-10ADS autophotography system. We also observed 100 200 cells in interphase and counted the number of cells showing a normal microtubule network. Our criteria for identifying cells that did not have a normal microtubule network were: (a) the presence of microtubule fibers or a layer wrapped around the nucleus; (b) aggregates and/or fragments of microtubules present in the cytosol. Each value obtained indicated the percentage of cells with a normal microtubule network. Similar experiments were performed twice.

\section{Results and Discussion}

\section{Cytotoxicities of 1, 2, 3 and 4 against V79 Cells}

The cytotoxicities of all four compounds in V79 cells were obtained by determining the relative plating efficiency when cells were treated with various concentrations of the drugs for 48 hours. As shown in Fig. 2, the $\mathrm{IC}_{50}$ (the concentration required for the $50 \%$ inhibition of colony formation) was $8 \mu \mathrm{M}$ for $\mathbf{1}, 0.9 \mu \mathrm{M}$ for $\mathbf{2}, 0.7 \mu \mathrm{M}$ for $\mathbf{3}$ and $1.2 \mu \mathrm{M}$ for 4 . Based on the above findings, 3 showed the highest cytotoxicity among the samples tested, revealing that $\mathbf{3}$ exhibits stronger cytotoxicity than $\mathbf{1}$, unlike the in vitro effect on microtubule proteins noted previously. Thus, we decided to examine the effects of these compounds on cellular microtubule networks.

\section{Effects of 1, 2, 3 and 4 on Cellular Microtubule Networks}

Next, the effects of all four compounds on cytoplasmic microtubules were investigated. Interphase cells in unsynchronized cultures were examined by the indirect immunofluorescence method employing anti- $\beta$-tubulin antibody. In untreated control cultures, the proportion of normal cells was $83 \%$. Cells treated with various concentrations $(1 \sim 30 \mu \mathrm{M})$ of $\mathbf{1}, \mathbf{2}, 3$ and 4 for 1 hour changed their morphology to a round shape with an accompanying abnormal architecture of the cytoplasmic microtubules (Fig. 3). The $\mathrm{EC}_{50}$ (the concentration required for the $50 \%$ disruption of the microtubule network) was $12 \mu \mathrm{M}$ for $1,5 \mu \mathrm{M}$ for $2,0.6 \mu \mathrm{M}$ for 3 and $15 \mu \mathrm{M}$ for 4 . These findings also clarified that $\mathbf{3}$ damaged most markedly the microtubules formation in the cytoskeleton similarly to the above results of the cytotoxicity.

The results of the relative cytotoxicities and effects on cellular microtubule networks are in good correlation, but they are not in agreement with effects on in vitro microtubule polymerization. It was observed in the cell system experiment that the cellular incorporation rates of these compounds were influenced by the substituents at 2 'position and that a higher incorporation rate was obtained with 3. This may be the reason for the incompatible results reported from in vitro and in vivo experiments mentioned above, with $\mathbf{3}$ possibly being taken up by cellular microtubule systems more efficiently. 


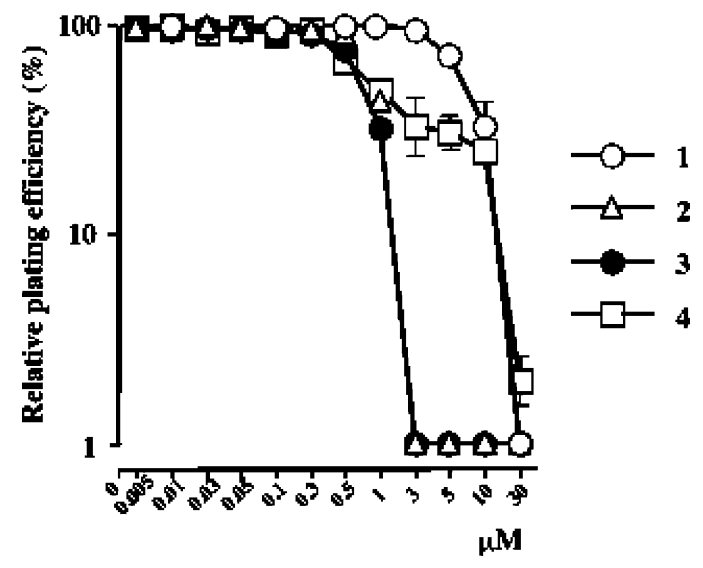

Fig. 2 Relative plating efficiencies of Chinese hamster V79 cells.

Cells were treated with griseofulvin derivatives $(\mathbf{1}, \mathbf{2}, \mathbf{3}$ and $\mathbf{4})$ for 48 hours at various concentrations.

Consequently, 3 may exhibit stronger effects on cancer cells than 1 because it interferes with microtubules similarly to the vinca alkaloids and taxanes, which are important chemotherapeutic agents for the treatment of cancer. From this experimental data, it is expected that the activity of $\mathbf{3}$ will be enhanced by structure modifications.

Acknowledgements I thank Messrs. M. Kurihara, S. Sagawa and A. Kuroda for their excellent technical assistance.

\section{References}

1. Huber FM. Antibiotics I, Mechanism of Action, Ed. Gottlieb D, Shaw PD, p. 181 (1967)

2. Sato Y, Oda T, Saito H. A novel biosynthetic study of griseofulvin by ${ }^{2} \mathrm{H}$ nuclear magnetic resonance: determination of deuterium incorporation from $\left[2-{ }^{2} \mathrm{H}_{3}\right]$-acetate by Penicillium urticae. Tetrahedron Lett 2695-2698 (1976)

3. Sato Y, Oda T, Urano S. Griseofulvin biosynthesis: new evidence of two acetate-dispositions in the ring A from ${ }^{13} \mathrm{C}$ nuclear magnetic resonance studies. Tetrahedron Lett 3971-3974 (1976)

4. Sato Y, Oda T, Saito H. Microbial transformation of dehydrogriseofulvin and griseofulvin ${ }^{2} \mathrm{H}$ NMR and mass spectrometric studies of stereochemical courses of microbial hydrogenation and hydroxylation. J Chem Soc Chem Commun 415-417 (1977)

5. Oda T, Sato Y. (+)-Griseofulvin with a cell-free system of Streptomyces cinereocrocatus. Chem Pharm Bull 33: 1077-1082 (1985)

6. Muehlbauer PA, Schuler MJ. Detection of numerical chromosomal aberrations by flow cytometry: a novel process for identifying aneugenic agents. Mutat Res 585: 156-169 (2005)

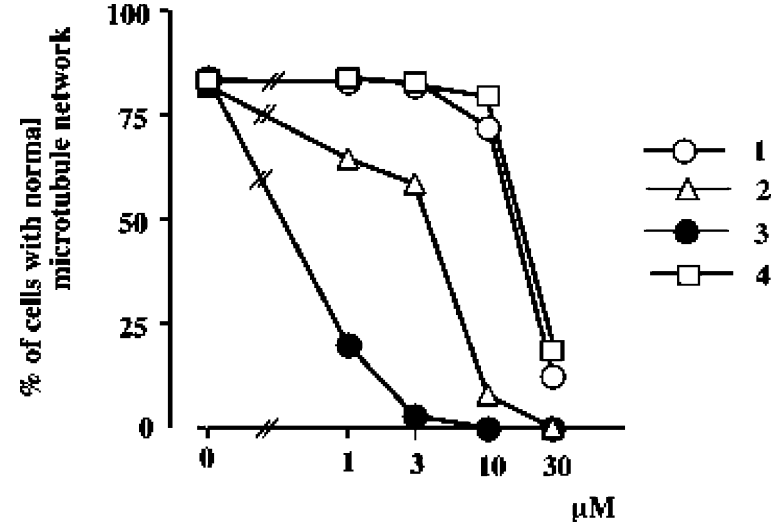

Fig. 3 Effects of griseofulvin derivatives (1, 2, 3 and $\mathbf{4}$ ) on the microtubule network in Chinese hamster V79 cells.

Cells were treated with various concentrations of griseofulvin derivatives $(\mathbf{1}, \mathbf{2}, \mathbf{3}$ and $\mathbf{4})$ for 1 hour; thereafter, the cells were stained and the number of cells with normal microtubule networks was counted, as described in the "Materials and Methods."

7. Panda D, Rathinasamy K, Santra MK, Wilson L. Kinetic suppression of microtubule dynamic instability by griseofulvin: implications for its possible use in the treatment of cancer. Proc Natl Acad Sci USA 102: 9878-9883 (2005)

8. Weisenberg RC. Microtubule formation in vitro in solutions containing low calcium concentrations. Science 177: 1104-1105 (1972)

9. Hoebeke J, Nijen GV. Quantitative turbidimetric assay for potency evaluation of colchicine-like drugs. Life Sci 17: 591-595 (1975)

10. Olmsted JB, Borisy GG. Characterization of microtubule assembly in porcine brain extracts by viscometry. Biochemistry 12: 4282-4289 (1973)

11. Sato Y, Saito Y, Shiratori Y, Syouda S, Hosoi J. The structure-activity relationship of griseofulvin and its derivatives with microtubules and microtubule proteins. Nippon Kagaku Kaishi 5: 746-754 (1981)

12. Oda T, Sato Y. Microbial transformation of $(+)-$ and (-)-2'-demethoxydehyudrogriseofulvin by Streptomyces cinereocrocatus. Chem Phar Bull 31: 1698-1701 (1983)

13. Sato Y, Oda T. Microbial transformation of $2^{\prime}$-propoxy analogs of (-)- and (+)-dehydrogriseofulvin and $(+)-2$-demethoxydehydrogriseofulvin by Streptomyces cinereocrocatus. Heterocycles 17: 171-176 (1982)

14. Oda T, Hashimoto H, Sato Y. Regio- and stereoselective hydrogenation of $2^{\prime}$-demethoxy-2'-methyldehydrogriseofulvin, a symmetrical substrate, to $(+)-2^{\prime}$-demethoxy2 '-methylgriseofulvin with a cell-free system of Streptomyces cinereocrocatus. Chem Pharm Bull 38: 525-528 (1990)

15. Sakakibara Y, Saito I, Ichinoseki K, Oda T, Kaneko M, Saito H, Kodama M, Sato Y. Effects of diethylstilbestrol and its methyl ethers on aneuploidy induction and microtubule distribution in Chinese hamster V79 cells. Mutation Res 263: 269-276 (1991) 